Original article

\title{
Simultaneous estimation of degree of crystallinity in combination drug product of abacavir, lamivudine and neverapine using X-ray powder diffraction technique
}

\author{
Pavan Kommavarapu*, Arthanareeswari Maruthapillai, Ravikiran Allada, \\ Kamaraj Palanisamy, Praveen Chappa \\ Department of Chemistry, SRM University, Kattankulathur 603203, Tamil Nadu, India
}

\section{A R T I C L E I N F O}

\section{Article history:}

Received 19 August 2013

Accepted 18 October 2013

Available online 14 November 2013

\section{Keywords:}

Crystallinity

Drug product

X-ray powder diffraction

Regressions statistics

Analysis of variance

\begin{abstract}
A B S T R A C T
Objective: In the present study, simultaneous determination of degree of crystallinity content in abacavir (ABC), lamivudine (3TC) and neverapine (NVP) from there combination drug product using X-ray powder diffraction (XRPD) technique is developed and validated.

Methods: The X-ray procedure for the identification and determination of the degree of crystallinity in $A B C, 3 T C$ and NVP drug product is developed and validated. It is based on the X-ray diffraction from crystalline region of the drug product. The characteristic peaks of the three drugs were characterized using XRPD.

Results: ABC, 3TC and NVP concentrations ranging from $70 \%$ to $130 \%$ in drug product were prepared and linearity in this concentration range is described. The \% coefficient of variation (\%CV) was found to be 0.9982 for $\mathrm{ABC}, 0.9978$ for $3 \mathrm{TC}$ and 0.9984 for NVP. The mean recoveries were found to be $100.3 \%$ for $\mathrm{ABC}$, 99.0\% for 3TC and $100.8 \%$ for NVP. Regressions statistics and Analysis of variance (ANOVA) table results were evaluated and found to be satisfactory.

Conclusions: The method has been applied to unknown mixtures of drug formulations and stability samples. The proposed method can be useful in the quality control of combination drug products.

Copyright (c) 2013, InPharm Association, Published by Reed Elsevier India Pvt. Ltd. All rights reserved.
\end{abstract}

\section{Introduction}

The managing of human immunodeficiency virus (HIV) includes the use of multiple antiretroviral, since single drug therapy becomes ineffective due to development of HIV resistant strains. According to treatment guidelines antiretroviral regimen should contain at least two nucleoside analogue reverse transcriptase inhibitors (NRTIs) and one non-nucleoside reverse transcriptase inhibitor (NNRTI) in a fixed dose combination. ${ }^{1,2}$ An example of such combination is ABC, 3TC and NVP, which is very efficient in the treatment of HIV. Abacavir (ABC) is a nucleoside reverse transcriptase inhibitor (NRTI) with activity against Human Immunodeficiency Virus Type 1 (HIV-1). ABC is phosphorylated to active metabolites that compete for incorporation into viral DNA. Intracellularly, $\mathrm{ABC}$ is converted by cellular enzymes to the active metabolite carbovir triphosphate, an analogue of deoxyguanosine-5'-triphosphate (dGTP). Chemically ABC is [(1S, 4R)-4-[2-

\footnotetext{
* Corresponding author. Tel.: +91 8106791313.

E-mail address: kommavarapu.pavan@gmail.com (P. Kommavarapu).
}

amino-6-(cyclopropylamino)-9H-purin-9-yl] cyclopent-2-en-1-yl] methanol. ${ }^{3-5}$ Lamivudine (3TC) is a nucleoside reverse transcriptase inhibitor (NRTI) with activity against Human Immunodeficiency Virus Type 1 (HIV-1) and hepatitis $B(\mathrm{HBV})$. 3TC is a synthetic nucleoside analogue and is phosphorylated intracellularly to its active $5^{\prime}$ triphosphate metabolite, lamivudine triphosphate (L-TP). Chemically 3TC is 4-amino-1-[(2R, 5S)-2-(hydroxymethyl)-1, 3-oxathiolan-5-yl]1, 2-dihydropyrimidin-2-one. ${ }^{6-8}$ Neverapine (NVP), is a nonnucleoside reverse transcriptase inhibitor (NNRTI) used to treat HIV-1 infection and AIDS. Neverapine falls in the non-nucleoside reverse transcriptase inhibitor (NNRTI) class of antiretrovirals. ${ }^{9}$ Chemically NVP is 11-cyclopropyl-4-methyl-5,11-dihydro-6H-dipyrido[3,2-b:2',3'-e][1,4]diazepin-6-one.

The objective of the present study is to describe an X-ray diffraction quantitative method for the simultaneous estimation of degree of crystallinity of ABC, 3TC and NVP in a triple composition drug product. The X-ray diffraction pattern consists of information about peak positions and intensity, which are indicative of the crystal structure, composition and symmetry of the contributing phase. The peak intensities reflect the total scattering from the each 
plane in the phase's crystal structure, and are directly dependent on the distribution of particular atoms in the structure. The diffraction intensity equation is summarized below:

$$
\begin{aligned}
I_{(\mathrm{hkl}) \alpha}= & \frac{I_{0} \lambda^{3}}{64 \pi r}\left(\frac{e^{2}}{m_{\mathrm{e}} c^{2}}\right)^{2} \frac{M_{(\mathrm{hkl})}}{V_{\alpha}^{2}}\left|F_{(\mathrm{hkl}) \alpha}\right|^{2} \\
& \left(\frac{1+\cos ^{2}(2 \theta) \cos ^{2}\left(2 \theta_{\mathrm{m}}\right)}{\sin ^{2} \theta \cos \theta}\right)_{\mathrm{hkl}} \frac{v_{\alpha}}{\mu_{\mathrm{s}}}
\end{aligned}
$$

where, $I_{(\mathrm{hkl}) \alpha}$ is the Intensity of reflection of hkl in phase $\alpha, I_{0}$ is the incident beam intensity, $r$ is the distance from specimen to detector, $\lambda$ is X-ray wavelength, $\left(e^{2} / m_{\mathrm{e}} c^{2}\right)^{2}$ is the square of classical electron radius, $\mu_{\mathrm{s}}$ is linear absorption coefficient of the specimen, $V_{\alpha}$ is volume fraction of phase $\alpha, M_{\mathrm{hkl}}$ is multiplicity of reflection hkl of phase $\alpha, v \alpha$ is volume of the unit cell of phase $\alpha, 2 \theta_{\mathrm{m}}$ is the diffraction angle of the monochromator and $F_{(\mathrm{hkl}) \alpha}$ is the structure factor for reflection hkl of phase $\alpha$ (i.e., the vector sum of scattering intensities of all atoms contributing to that reflection). ${ }^{10}$

Methods have been developed which use peak intensities for quantitative analysis of diffraction data. Some of the quantitative Methods based on Intensity Ratios of diffraction data are Absorption-Diffraction Method, Method of Standard Additions, Internal Standard Method, Reference Intensity Ratio Methods and Full-Pattern Analysis - the Rietveld Method. ${ }^{11,12}$ In the present study regression analysis is carried out to establish the equation that best describes the linear relationship between instrument response $(y)$ and analyte level $(x)$. The relationship is given by the equation of the line, $y=m x+c$ where $m$ is the gradient of the line and $c$ is its intercept with the $y$-axis. Linear regression establishes the values of $m$ and $c$, which best describes the relationship between the data sets. The equations for calculating $m$ and $c$ are given as follows:

$m=\frac{\sum_{i=1}^{n}\left\{\left(x_{i}-\bar{x}\right)\left(y_{i}-\bar{y}\right)\right\}}{\sum_{i=1}^{n}\left(x_{i}-\bar{x}\right)^{2}}$

$c=\bar{y}-m \bar{x}$

The correlation coefficient $(R)$ is a measure of the strength of the degree of correlation between the $y$ and $x$ values. $R$ can take any value between +1 and -1 ; the closer it is to 1 , the stronger the correlation. $R$ is given by the following equation.

$R=\frac{\sum_{i=1}^{n}\left\{\left(x_{i}-\bar{x}\right)\left(y_{i}-\bar{y}\right)\right\}}{\left\{\left[\sum_{i=1}^{n}\left(x_{i}-\bar{x}\right)^{2}\right]\left[\left[\sum_{i=1}^{n}\left(y_{i}-\bar{y}\right)^{2}\right]\right]\right\}^{1 / 2}}$

where $x_{\mathrm{i}}$ and $y_{\mathrm{i}}$ are the value on $x$-axis and $y$-axis, $\bar{x}$ and $\bar{y}$ are the mean of $x_{\mathrm{i}}$ and $y_{\mathrm{i}}$ values and $n$ is the number of calibration points. The parameters related to $R$ are $R^{2}$ and adjusted $R^{2} \cdot R^{2}$ is often used to describe the fraction of the total variance in the data which is contributed by the line that has been fitted. Ideally, if there is a good linear relation, the majority of variability can be accounted for by the fitted line. $R^{2}$ value is close to 1 . The adjusted $R^{2}$ value is interpreted in the same way as $R^{2}$ but is always lower. It is useful for assessing the effect of adding additional terms to the equation of the fitted line (e.g., if a quadratic fit is used instead of a linear fit). The $R^{2}$ value always increases on the addition of an extra term to the equation, but this does not mean that the extended equation is necessarily a better fit of the data. The adjusted $R^{2}$ value is more useful in such cases as it takes account of the reduction in the degrees of freedom, which occurs each time an additional term is added to the equation of the line and therefore does not automatically increase on addition of extra terms. Another important parameter in regression analysis is Residual standard deviation (or standard error), which is a statistical measure of the deviation of the data from the fitted regression line. It is calculated using the following equation:

$s(r)=\sqrt{\frac{\sum_{i=1}^{n}\left(y_{i}-\widehat{y}_{i}\right)^{2}}{n-2}}$

where $y_{\mathrm{i}}$ is the observed value of $y$ for a given value of $x_{\mathrm{i}}, \hat{y}_{\mathrm{i}}$ is the value of $y$ predicted by the equation of the calibration line for a given value of $x_{\mathrm{i}}$ and $n$ is the number of calibration points. Analysis of variance (ANOVA) table for the regression is produced in regression analysis. In ANOVA table the sum of squares terms (SS) represent different sources of variability in the calibration data. The regression term represents the variability in the data that can be accounted for by the fitted regression line. The residual term is the sum of the squared residuals. Each mean square (MS) term is simply the sum of squares term divided by its degrees of freedom. The F value is the ratio of the regression MS term to the residual MS term. The significance $F$ value represents the probability of obtaining the results in the ANOVA table if there is no correlation between $y$ and $x$ values, i.e., obtaining the results by chance. A small value indicates that the results were unlikely to have happened by chance, indicating that it is highly likely that there is a strong relationship between the $y$ and $x$ values. For a calibration curve to be of any use the significance $F$ value should be extremely small. This value is also known as the $p$-value. ${ }^{13-17}$

To our knowledge, based on extensive review, no efforts have been made in published literature to determine the degree of crystallinity simultaneously in ABC, 3TC and NVP combination drug product, determination of regression statistics and evaluation of ANOVA table parameters.

\section{Experimental}

The three drugs ABC, 3TC and NVP were conformed using PXRD. The required concentrations ranging between $70 \%$ and $130 \%$ were prepared accurately using high Sensitive Microbalance (Mettler Toledo XP2U, Max $\left.2.1 \mathrm{~g}-\mathrm{Min}-1^{\circ} \mu \mathrm{g}\right)$.

\subsection{PXRD analysis}

Diffraction patterns were collected using Bruker D8 advance X-ray diffractometer with $\mathrm{Cu}$ anode and Lynx eye detector. ABC, 3TC and NVP were scanned from $3^{\circ} 2 \theta$ to $45^{\circ} 2 \theta$, with step size $0.01^{\circ} 2 \theta$ and time per step of $0.1 \mathrm{~s}$ for the identification purpose. After selecting the non-interfering peak, the patterns of spiked standards were collected from $12^{\circ} 2 \theta$ to $21^{\circ} 2 \theta$, with step size $0.01^{\circ} 2 \theta$ and time per step of $0.72 \mathrm{~s}$. The instrument was operated at $40 \mathrm{kV}$ generator voltage and $40 \mathrm{~mA}$ generator current. Variable divergent slit and Anti scattering slit were used of $\mathrm{V}_{20} \mathrm{~mm}$, Nickel filter was used in secondary beam path. Eva software was used for data processing and evaluation.

Approved raw material of ABC, 3TC and NVP assigned against primary reference standard is used for the study. The chemical structures are represented in Fig. 1. Spiked standard concentrations were prepared by geometrical mixing of $\mathrm{ABC}$, 3TC, NVP and placebo. Average weight of tablet is $394 \mathrm{mg}$, which contains about $69 \mathrm{mg}$ of abacavir sulphate which is equivalent to $60 \mathrm{mg}$ of abacavir, $30 \mathrm{mg}$ of lamivudine and $50 \mathrm{mg}$ of neverapine. Eleven standard blend mixtures were prepared whose average weight is calculated about $500 \mathrm{mg}$ for convenient packing in sample holders. The ratio of ABC, 3TC and NVP taken for the method development and quantitative determination of crystallinity in final drug product are tabulated in Table 1. 
<smiles>Nc1nc(NC2CC2)c2ncn([C@H]3C=C[C@H](CO)C3)c2n1</smiles>

a<smiles>Nc1ccn([C@H]2CS[C@@H](CO)O2)c(=O)n1</smiles>

b<smiles>Cc1ccnc2c1NC(=O)c1cccnc1N2C1CC1</smiles>

C

Fig. 1. Chemical structure of Abacavir (a), Lamivudine (b) and Neverapine (c)

\% Concentration of ABC, 3TC and NVP in Standard-1 is 69.9, 130.1 and 129.9; Standard-2 is 75.1, 125.3 and 125.2; Standard-3 is 80.1, 120.3 and 120.1 ; Standard-4 is $84.7,114.9$ and 115.2 ; Standard-5 is 90.1, 110.2 and 109.7; Standard-6 is 95.3, 105.1 and 105.3; Standard7 is 100.4, 100.1 and 100.2; Standard-8 is 110.1, 90.1 and 90.3; Standard-9 is 115.1, 85.2 and 85.1 ; Standard-10 is $120.2,80.1$ and 80.2 and Standard-11 is $129.8,70.1$ and 70.3 .

\subsection{Method validation}

The proposed method was validated with respect to selectivity, linearity, precision and recovery. ${ }^{18}$

\subsubsection{Selectivity}

The selectivity of the method was determined to make sure that ABC, 3TC and NVP characteristics peaks were well separated and free from interference of Placebo in the drug product.

\subsection{Linearity}

Standard sample preparations with concentration range of $70 \%-130 \%$ were prepared by spiking ABC, 3TC and NVP in placebo. Standard calibration curve was constructed by plotting the peak areas of $\mathrm{ABC}, 3 \mathrm{TC}$ and NVP against the corresponding

Table 1

Weights of ABC, 3TC, NVP \& Placebo taken for the preparation of standard blend mixtures.

\begin{tabular}{lcclll}
\hline $\begin{array}{l}\text { Standard blend } \\
\text { mixtures }\end{array}$ & $\begin{array}{l}\text { ABC } \\
(\mathrm{mg})\end{array}$ & $\begin{array}{l}\text { 3TC } \\
(\mathrm{mg})\end{array}$ & $\begin{array}{l}\text { NVP } \\
(\mathrm{mg})\end{array}$ & $\begin{array}{l}\text { Placebo } \\
(\mathrm{mg})\end{array}$ & $\begin{array}{l}\text { Total weight of } \\
\text { the blend }(\mathrm{mg})\end{array}$ \\
\hline Standard-1 & 61.51 & 49.44 & 81.84 & 307.29 & 500.08 \\
Standard-2 & 66.09 & 47.61 & 78.88 & 307.52 & 500.10 \\
Standard-3 & 70.49 & 45.71 & 75.66 & 308.19 & 500.06 \\
Standard-4 & 74.54 & 43.66 & 72.58 & 308.50 & 499.27 \\
Standard-5 & 79.29 & 41.88 & 69.11 & 309.90 & 500.18 \\
Standard-6 & 83.86 & 39.94 & 66.34 & 310.22 & 500.36 \\
Standard-7 & 88.35 & 38.04 & 63.13 & 310.42 & 499.94 \\
Standard-8 & 96.89 & 34.24 & 56.89 & 311.85 & 499.87 \\
Standard-9 & 101.29 & 32.38 & 53.61 & 311.98 & 499.26 \\
Standard-10 & 105.78 & 30.44 & 50.53 & 312.54 & 499.28 \\
Standard-11 & 114.22 & 26.64 & 44.29 & 313.94 & 499.09 \\
\hline
\end{tabular}

concentrations of standard samples. Linear regression equation and correlation coefficient were determined.

\subsection{Precision}

The precision of this analytical method is expressed as the relative standard deviation of a series of measurements. Spiked standard concentrations of ABC, 3TC and NVP at 95\%, 105\% and 110\% concentration levels were prepared and scanned for three times.

\subsection{Recovery}

Three concentrations of ABC, 3TC and NVP in placebo i.e., 95\%, $105 \%$ and $110 \%$ were prepared and three replicates of each concentration are scanned.

\section{Results and discussion}

The three drugs ABC, 3TC and NVP were identified and characterised using PXRD and the overlay of the diffractogram of the drugs are represented in Fig. 2 . The characteristics peaks with their corresponding intensity $\%$ of the three drugs are represented in

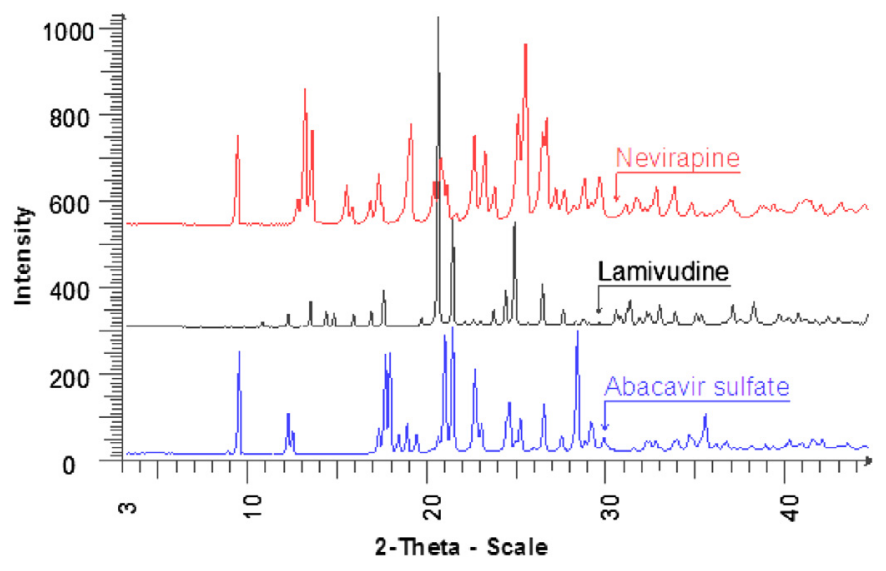

Fig. 2. Overlaid diffractrograms of ABC, 3TC and NVP. 
Table 2

Characteristics peaks of $\mathrm{ABC}$, 3TC and NVP.

\begin{tabular}{|c|c|c|c|c|c|}
\hline \multicolumn{2}{|l|}{ Abacavir } & \multicolumn{2}{|l|}{ Lamivudine } & \multicolumn{2}{|l|}{ Neverapine } \\
\hline Angle $\left(2 \theta^{\circ}\right)$ & Intensity \% & Angle $\left(2 \theta^{\circ}\right)$ & Intensity \% & Angle $\left(2 \theta^{\circ}\right)$ & Intensity \% \\
\hline 8.736 & 5.0 & 10.666 & 2.2 & 9.285 & 50.3 \\
\hline 9.37 & 81.9 & 12.143 & 4.4 & 10.295 & 2.7 \\
\hline 12.138 & 34.2 & 13.396 & 8.6 & 12.662 & 15.6 \\
\hline 12.385 & 19.6 & 14.279 & 5.4 & 13.096 & 75.6 \\
\hline 17.253 & 21.9 & 14.731 & 4.8 & 13.495 & 53.2 \\
\hline 17.628 & 80.1 & 15.836 & 4.4 & 15.439 & 23.3 \\
\hline 17.884 & 80.4 & 16.816 & 5.4 & 15.745 & 11.7 \\
\hline 18.381 & 17.9 & 17.529 & 11.7 & 16.773 & 14.8 \\
\hline 18.859 & 26.2 & 19.669 & 3.3 & 17.255 & 28.9 \\
\hline 19.392 & 17.5 & 20.612 & 100.0 & 17.441 & 13.8 \\
\hline 20.245 & 6.3 & 21.426 & 33.2 & 18.523 & 7.5 \\
\hline 20.603 & 15.6 & 22.124 & 2.3 & 18.937 & 41.4 \\
\hline 20.988 & 94.3 & 22.597 & 2.7 & 19.072 & 56.6 \\
\hline 21.425 & 100.0 & 23.005 & 2.8 & 20.372 & 25.1 \\
\hline 22.685 & 68.1 & 23.746 & 6.0 & 20.754 & 38.0 \\
\hline 23.053 & 25.6 & 24.418 & 11.7 & 20.878 & 30.5 \\
\hline 24.492 & 30.5 & 24.922 & 32.5 & 21.106 & 23.7 \\
\hline 24.635 & 42.5 & 25.683 & 2.0 & 21.624 & 8.1 \\
\hline 24.985 & 13.0 & 26.491 & 13.6 & 22.658 & 50.4 \\
\hline 25.257 & 29.6 & 27.669 & 5.9 & 23.258 & 41.4 \\
\hline 26.009 & 7.3 & 28.39 & 2.2 & 23.787 & 22.4 \\
\hline 26.585 & 40.8 & 28.771 & 2.8 & 25.126 & 61.3 \\
\hline 27.586 & 16.4 & 28.875 & 2.8 & 25.544 & 100.0 \\
\hline 28.471 & 98.0 & 29.096 & 1.8 & 26.506 & 51.7 \\
\hline 28.885 & 12.5 & 29.697 & 1.8 & 26.738 & 60.0 \\
\hline 29.244 & 27.1 & 30.656 & 5.7 & 27.235 & 21.4 \\
\hline 29.976 & 15.6 & 30.885 & 4.2 & 27.733 & 20.7 \\
\hline 30.203 & 8.5 & 31.297 & 6.3 & 28.265 & 12.3 \\
\hline 30.467 & 6.1 & 31.442 & 8.6 & 28.874 & 26.9 \\
\hline 31.652 & 6.9 & 31.964 & 3.4 & 29.248 & 14.2 \\
\hline
\end{tabular}

Table 2. Form this information we can select non-interfering peaks of $A B C$, 3TC and NVP which are used in the creation of calibration curves.

The standard calibration curves (Fig. 3.) were constructed by plotting spiked standard preparations against Net area (counts per seconds $(\mathrm{Cps}) \times 2 \theta^{\circ}$ ). Overlaid diffractograms of different spiked standard preparations are represented in Fig. 4. The standard calibration curve for ABC, 3TC and NVP was linear over a concentration range of $70 \%-130 \%$. The correlation coefficient $\left(R^{2}\right)$ was 0.9982 for ABC, 0.9978 for 3TC and 0.9984 for NVP, which are well within acceptable limit. ${ }^{18}$ Linear regression equations of ABC, 3TC and NVP were found to be $y=0.0578 x-0.1, y=0.219 x-14.511$ and $y=0.0832 x-4.9302$ respectively.

Three standard preparations at $95 \%, 105 \%$ and $110 \%$ level were prepared and measurements are collected for three replicates and

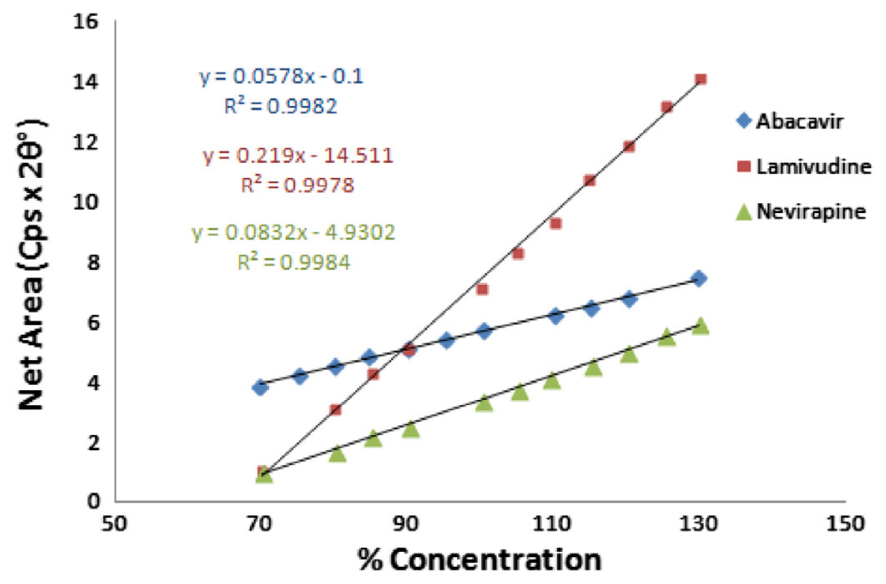

Fig. 3. The standard calibration curves for ABC, $3 T C$ and NVP.

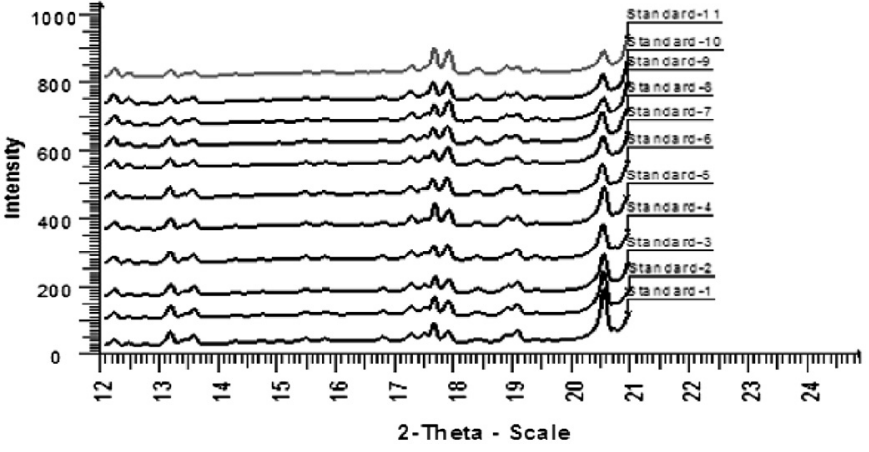

Fig. 4. Overlaid diffractrograms of Spiked standard preparations of ABC, 3TC and NVP.

Table 3

Precision, Accuracy and Reproducibility data.

\begin{tabular}{lcccccc}
\hline \multirow{2}{*}{$\begin{array}{l}\text { Actual } \\
\text { weight \% }\end{array}$} & \multicolumn{6}{l}{ Calculated weight \% } \\
\cline { 2 - 7 } & ABC & \% Recovery & 3TC & \% Recovery & NVP & \% Recovery \\
\hline 95 & 95.3 & 100.3 & 94.5 & 99.5 & 94.7 & 99.7 \\
& 95.1 & 100.1 & 94.9 & 99.9 & 94.9 & 99.9 \\
& 95.4 & 100.4 & 94.8 & 99.8 & 95.2 & 100.2 \\
Mean & 95.3 & & 94.7 & & 94.9 & \\
\%RSD & 0.16 & & 0.22 & & 0.27 & \\
105 & 103.2 & 98.3 & 104.9 & 99.9 & 106.4 & 101.3 \\
& 106.1 & 101.0 & 102.6 & 97.7 & 105.7 & 100.7 \\
& 104.7 & 99.7 & 103.8 & 98.9 & 106.2 & 101.1 \\
Mean & 104.7 & & 103.8 & & 106.1 & \\
\%RSD & 1.39 & & 1.11 & & 0.34 & \\
110 & 111.7 & 101.5 & 106.9 & 97.2 & 112.7 & 102.5 \\
& 113.6 & 103.3 & 109.1 & 99.2 & 111.9 & 101.7 \\
& 107.5 & 97.7 & 108.7 & 98.8 & 110.2 & 100.2 \\
Mean & 110.9 & & 108.2 & & 111.6 & \\
\%RSD & 2.81 & & 1.08 & & 1.14 & \\
\hline
\end{tabular}

corresponding peaks of $\mathrm{ABC}$, 3TC and NVP were integrated for the evaluation of precision, recovery, accuracy and reproducibility (Table 3). The mean recovery values of $100.3 \%$ for ABC, $99.0 \%$ for $3 T C$ and $100.8 \%$ for NVP were obtained. Average recovery for each drug was above $99 \%$. The low \%RSD value obtained by the triplicate measurement of each of the concentration of ABC, 3TC and NVP indicates that the method is precise and reproducible.

Regression statistics are evaluated for prepared spiked standard concentrations versus Net area obtained. Parameters like multiple $R, R^{2}$, Adjusted $R^{2}$ and Standard error are assessed. The correlation coefficient $R$ (Multiple $R$ ), $R^{2}$ and Adjusted $R^{2}$ values for ABC, 3TC and NVP are tabulated in Table 4.

The correlation coefficient $(R)$ for ABC, 3TC and NVP is $0.999083539,0.998905611$ and 0.99918471 respectively indicating the stronger relationship. $R^{2}$ and adjusted $R^{2}$ are the parameters related to $R$ and they provide us the information about fraction of the total variance in data added by the fitted line. Adjusted $R^{2}$ value is always lower than $R^{2}$ value.

Table 4

Regression statistics of ABC, 3TC and NVP.

\begin{tabular}{llll}
\hline Regression Statistics & & & \\
\hline & ABC & 3TC & NVP \\
\hline Multiple $R$ & 0.999083539 & 0.998905611 & 0.99918471 \\
$R^{2}$ & 0.998167918 & 0.997812419 & 0.998375602 \\
Adjusted $R^{2}$ & 0.997964354 & 0.997569355 & 0.998195114 \\
Standard Error & 0.051067604 & 0.211444399 & 0.068830267 \\
Observations & 11 & 11 & 11 \\
\hline
\end{tabular}


Table 5

ANOVA table for the regression of ABV, 3TC and NVP.

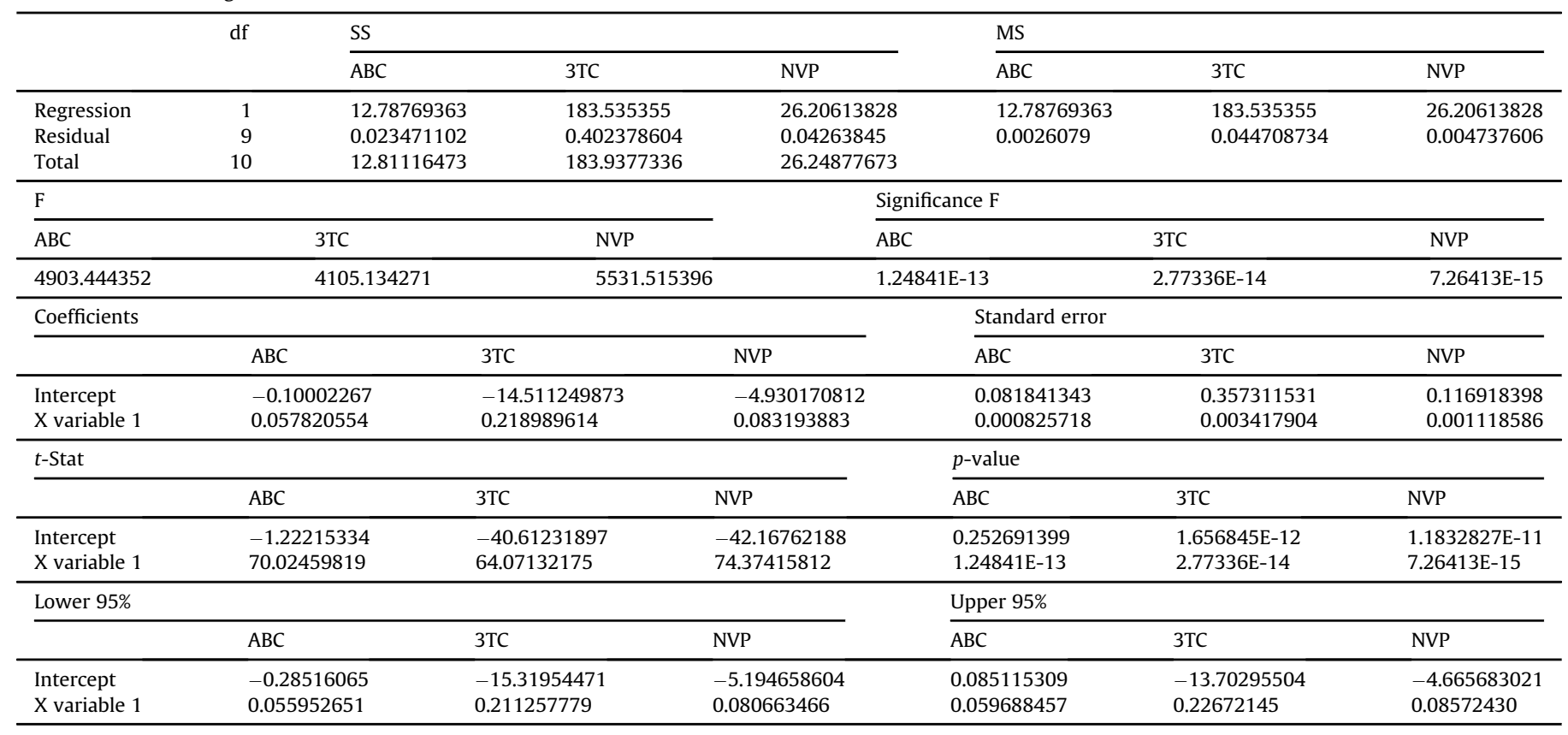

Table 6

\% Crystallinity determination of unknown samples.

\begin{tabular}{|c|c|c|c|c|c|c|c|}
\hline \multicolumn{8}{|c|}{ \% Crystallinity } \\
\hline & Stability condition & $\mathrm{ABC}$ & Net Area $\left(\mathrm{Cps} \times 2 \theta^{\circ}\right)$ & 3TC & Net Area $\left(\mathrm{Cps} \times 2 \theta^{\circ}\right)$ & NVP & Net Area $\left(\operatorname{Cps} \times 2 \theta^{\circ}\right)$ \\
\hline Sample 1 & Initial & 99.4 & 5.645 & 99.5 & 7.280 & 98.7 & 3.282 \\
\hline Sample 2 & $40{ }^{\circ} \mathrm{C} / 75 \%$ RH 1 Month & 98.7 & 5.605 & 98.4 & 7.039 & 98.3 & 3.248 \\
\hline Sample 3 & $40{ }^{\circ} \mathrm{C} / 75 \%$ RH 2 Months & 98.2 & 5.576 & 97.7 & 6.885 & 98.0 & 3.223 \\
\hline Sample 4 & $25^{\circ} \mathrm{C} / 60 \%$ RH 1 Month & 99.6 & 5.657 & 99.1 & 7.192 & 99.3 & 3.332 \\
\hline Sample 5 & $25^{\circ} \mathrm{C} / 60 \%$ RH 2 Months & 99.0 & 5.622 & 98.5 & 7.061 & 98.8 & 3.290 \\
\hline
\end{tabular}

Analysis of variance (ANOVA) table for the regression is constructed and is represented in Table 5 . The sum of squares terms (SS) and the regression term represents different sources of variability in the calibration data that can be accounted by the fitted regression line. The SS values for ABC, 3TC and NVP are 12.78769363, 183.535355 and 26.20613828 respectively. Since these values are large we conclude that there is a good linear relationship and the fitted line will describe majority of variability in response with concentration and this is also confirmed by the low residual values i.e., 0.023471102 for ABC, 0.402378604 for 3TC and 0.04263845 for NVP. The mean square term is the ratio of SS term to its degree of freedom ( $d f$ ) and the F value (which is the ratio of the regression MS term to the residual MS term) for ABC, 3TC and NVP are found to be 4903.444352, 4105.134271 and 5531.515396
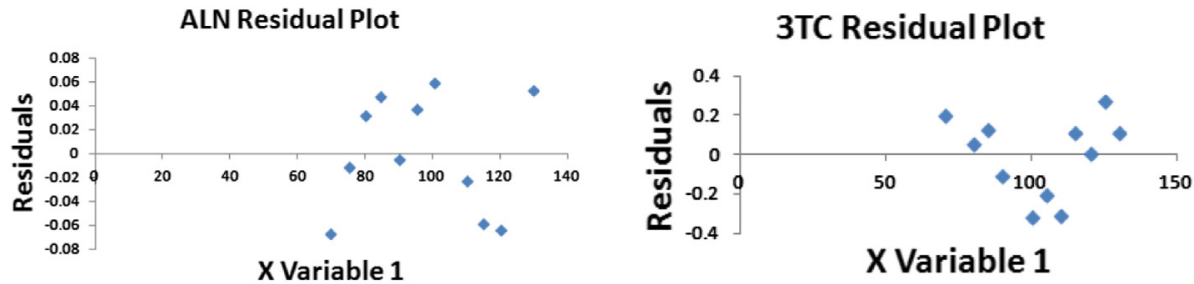

NVP Residual Plot

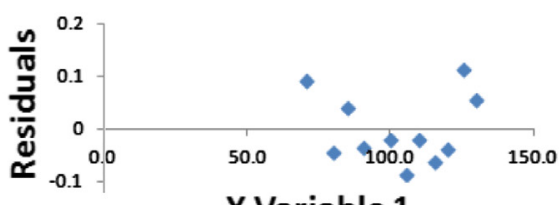

$\mathrm{X}$ Variable 1

Fig. 5. Residual plots of $A B C, 3 T C$ and NVP. 
respectively. The larger $\mathrm{F}$ values and the larger values of regression MS term compared to residual MS term indicate the good linear relationship. The significance $\mathrm{F}$ values ( $p$-values) for ABC, 3TC and NVP are found to be $1.24841 \mathrm{E}-13,2.77336 \mathrm{E}-14$ and $7.26413 \mathrm{E}-15$ respectively. The extreme small significance $F$ values for $A B C$, 3TC and NVP indicate that the results were unlikely to have happened by chance representing the strong relationship between data values. The standard error for each coefficient of ABC, 3TC and NVP are found to be $0.000825718,0.003417904$ and 0.001118586 respectively. The lower and upper confidence limits for the gradient and intercept at confidence level 95\% for ABC, 3TC and NVP are given in Table 5. These represent the extremes of the values that the gradient and intercept could take. Since the $t$-stat value is larger number and $p$-value greater than 0.05 for regression at $95 \%$ confidence level we can say that they are statistically significantly different from zero.

In order to assess the validity of the developed XRPD method it has been applied in the determination of crystalline content of drug formulation of unknown compositions initially and kept at different stability conditions. The results are evaluated from their respective calibration models. The results obtained are tabulated in Table 6. From the results obtained we conclude that the proposed method can be applied easily to analyse large number of samples to determine crystallinity content of drug product. Ideal residual plots are obtained for ABC, 3TC and NVP and are scattered randomly around zero Fig. 5.

\section{Conclusion}

Powder X-ray diffraction can be a viable method to monitor \% crystallinity stability of the active pharmaceutical ingredient in tablet formulations. Here it is shown that XRD measurements of the drug formulations under study were able to detect crystallinity content truly. Regression statistics and ANOVA table results demonstrates the adequacy of the method.

\section{Conflicts of interest}

All authors have none to declare.

\section{References}

1. Peter KQ. HIV drug resistance and the advent of integrase inhibitors. Curr Infect Dis Rep. 2013;15:85-100.

2. Gallant JE. Initial therapy of HIV infection. J Clin Virol. 2002;25:317-333.

3. Clercq DE. New anti-HIV agents and targets. Med Res Rev. 2002;22:531-565.

4. Lewis RJ, Tsai FT, Wigley DB. Molecular mechanisms of drug inhibition of DNA gyrase. Bioessays. 1996;18:661-671.

5. Yuen GJ, Weller S, Pakes GE. A review of the pharmacokinetics of abacavir. Clin Pharmacokinet. 2008;47:351-371.

6. Overington JP, Al-Lazikani B, Hopkins AL. How many drug targets are there? Nat Rev Drug Discov. 2006;5:993-996.

7. Imming P, Sinning C, Meyer A. Drugs, their targets and the nature and number of drug targets. Nat Rev Drug Discov. 2006;5:821-834.

8. Fox Z, Dragsted UB, Gerstoft J, et al. A randomized trial to evaluate continuation versus discontinuation of lamivudine in individuals failing a lamivudinecontaining regimen: the COLATE trial. Antivir Ther. 2006;11:761-770.

9. Patel SS, Benfield P. New drug profile: nevirapine. Clin Immunotherapeutics. 1996;6:307-317.

10. James RC. Introduction to X-ray Powder Diffraction. 2012. EPS400-002.

11. Jenkins R, Snyder RL. Quantitative Analysis, in Introduction to X-ray Powder Diffractometry. vol. 138. Hoboken, NJ, USA: John Wiley \& Sons, Inc.; 1996. http://dx.doi.org/10.1002/9781118520994.ch13.

12. Bish DL, Howard SA. Quantitative phase analysis using the Rietveld method. J Appl Crystallography. 1988:86-91.

13. Ronald AF. Statistical Methods for Research Workers. 12 ed. Edinburgh: Oliver and Boyd; 1954. ISBN: 0-05-002170-2.

14. Draper NR, Smith H. Applied Regression Analysis. 3rd ed. John Wiley; 1998. ISBN: 0-471-17082-8.

15. Bedson P, Mike S. The development and application of guidance on equipment qualification of analytical instruments. Accred Qual Assur. 1996:265-274.

16. Miller JN, Miller JC. Statistics and Chemometrics for Analytical Chemistry. 4th ed 2000. ISBN: 0-130-22888-5.

17. Barwick VJ. Preparation of Calibration Curves: A Guide to Best Practice. 2003 LGCVAM2003032.

18. ICH. International Conference on Harmonisation (ICH) of technical requirements for registration of pharmaceuticals for human use: harmonised tripartite guideline on validation of analytical procedures. In: Methodology, Recommended for Adoption at Step 4 of the ICH Process on by the ICH Steering Committee. Switzerland: IFPMA; 1996. 